\title{
Synovitis of small joints: sonographic guided diagnostic and therapeutic approach
}

\author{
Walter Grassi, Gianni Lamanna, Antonella Farina, Claudio Cervini
}

\begin{abstract}
Objective-The aim of this pictorial essay is to describe the sonographic guided approach to investigation and local injection therapy of a small joint in a patient with psoriatic arthritis (PA).

Methods-Sonographic pictures are obtained using a high frequency ultrasonography apparatus equipped with a $13-\mathrm{MHz}$ transducer.
\end{abstract}

Results-Ultrasonography allows a careful morphostructural assessment of soft tissue involvement in PA patients. Sonographic findings include joint cavity widening, capsular thickening, synovial proliferation, synovial fluid changes, tendon sheath widening. Ultrasound guided placement of the needle within the joint and injection of corticosteroid under sonographic control can be easily performed.

Conclusions-High frequency ultrasonography is a quick and safe procedure that

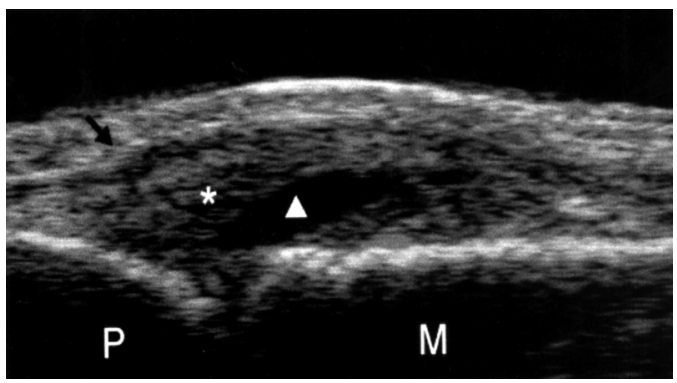

Figure 1 Longitudinal dorsal scan showing a clearly evident space widening of the MCP joint, synovial fluid, and synovial proliferation. Black arrow = extensor tendon; $\star$ = synovial proliferation; white triangle = synovial fluid; $P$ =proximal phalanx; $M=$ metacarpal bone.

Department of Rheumatology, University of Ancona, Italy

Correspondence to: Dr W Grassi, Clinica Reumatologica, Università degli Studi di Ancona, Ospedale A Murri, Via dei Colli, 52, I-60035 Jesi (Ancona), Italy.

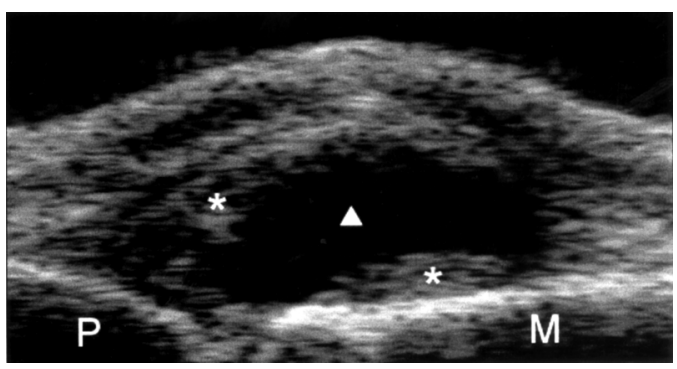

Figure 2 Longitudinal dorsal scan with the finger in passive hyperextension confirms that the mildly echogenic material within the joint space $\left(^{\star}\right)$ can be regarded as pannus. White triangle $=$ synovial fluid; $P=$ proximal phalanx; $M=$ metacarpal bone. allows a useful diagnostic and therapeutic approach in patients with arthritis of small joints.

(Ann Rheum Dis 1999;58:595-597)

Ultrasonography (US) with very high frequency transducer (13-20 MHz) shows excellent image quality for small joints and tendons. ${ }^{1-5}$ Patients with acute or chronic arthritis can have rapid, cheap, non-invasive and accurate evaluation of the anatomical changes occurring in a painful joint.

The purpose of this essay is to illustrate sonographic findings obtained in a metacarpophalangeal (MCP) joint of a patient with psoriatic arthritis (PA) during a conventional sonographic guided diagnostic and therapeutic approach. These pictures are a representative example of the useful application of US in daily rheumatology practice. ${ }^{6}$

\section{Clinical history}

The patient is a 45 year old woman who presents with a two year history of PA. She has a clinically relevant involvement of the third (MCP) joint of the right hand characterised by joint swelling and pain that is exacerbated by activity.

\section{Methods}

Images are obtained using a real time ultrasound equipment (AU4-idea; EsaOte Biomedica, Genoa, Italy) equipped with a $13 \mathrm{MHz}$ linear transducer. The standard technique for US of the MCP joints includes longitudinal and transverse scans both on the dorsal and volar planes.

Results and Discussion

A longitudinal dorsal scan of the MCP joint shows a clearly evident joint space widening (fig 1). The echogenicity of the joint space is characterised by the presence of both echoic and anechoic areas. The homogeneous anechoic and sharply defined area is the typical sonographic feature of synovial fluid. Conversely, echoic areas indicate synovial proliferation. This can be confirmed by dynamic examination of the same area. Figure 2 shows the same view of the joint of figure 1 after active hyperextension of the joint. The echoic areas are still clearly detectable and appear as 


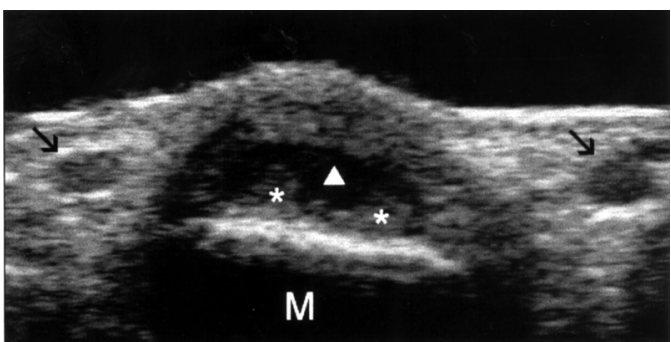

Figure 3 Transverse dorsal scan. Any doubt concerning the presence of proliferative synovitis is removed by a dorsal transverse view showing two demarcated areas of synovial hypertrophy $\left(^{*}\right)$. Black arrow = common palmar digital artery; white triangle = synovial fluid; $M=$ metacarpal bone.
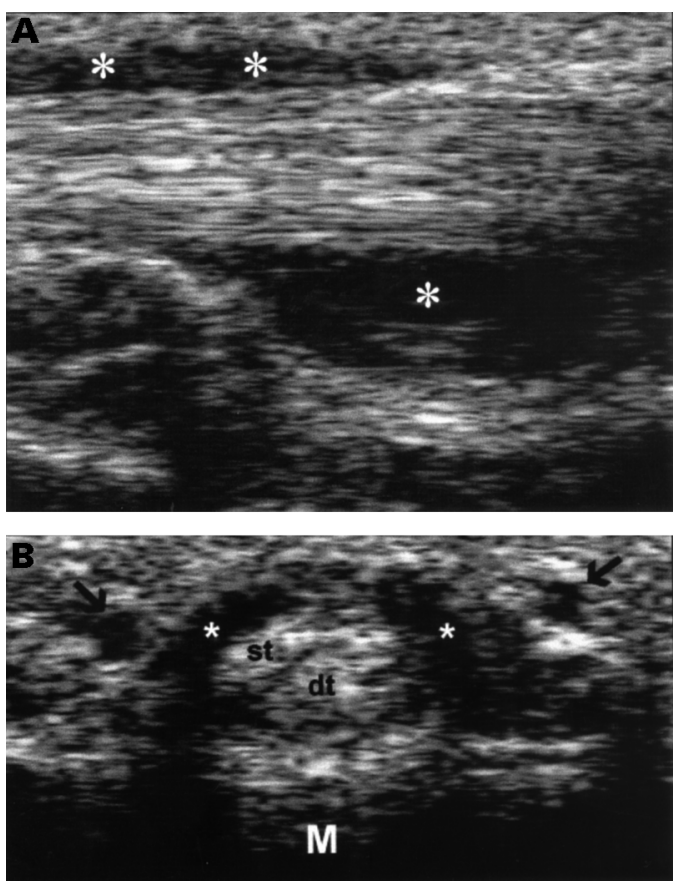

Figure 4 (A) Longitudinal sonogram of the MCP joint along the finger flexor tendons. This view allows identification of a clearly evident tendon sheath widening ${ }^{\star}{ }^{*}$. (B) Transverse sonogram of the same area confirms the presence of a homogeneous anechoic widening of the tendon sheath ( $\left.{ }^{\star}\right) . M=$ metacarpal bone; st = superficial flexor tendon; $d t=$ deep flexor tendon; black arrows = common palmar digital artery.

circumscribed sharply defined capsular thickening. Proteinaceous material within the joint space may mimic the echotexture of synovial hypertrophy. Compression of the joint by the transducer and/or passive and active movements of the joint do not change the sonographic features if the echoes are generated by synovial pannus. Proteinaceous material, on the contrary, changes its distribution within the joint and generates different pictures after mechanical stress of the joint. In this case, the clearly defined borders between the echoic and anechoic areas and the lack of change of the distribution of the echoes indicate the coexistence of both proliferative synovitis of the MCP joint and small effusion. Transverse views confirm the presence of both fluid and proliferative synovitis with polypoid aspect (fig 3).

Volar views confirm the presence of joint space widening and show the involvement of finger flexor tendons (tendon sheath widening) (fig $4 \mathrm{~A}, \mathrm{~B}$ ).

After a careful assessment of the MCP joint, a sonographic guided therapeutic injection of corticosteroids was decided. The transducer was placed along the line of the extensor tendon (longitudinal dorsal view). The entry point of the needle is about $1 \mathrm{~cm}$ distal to the centre of the joint space on the medial side of the extensor tendon.

The inclination of the needle is almost parallel to the skin surface to ensure the best visualisation of the different steps of the injection. When the tip of the needle is in the best position (at the centre of the anechoic area corresponding to synovial fluid), the corticosteroid is injected. Triamcinolone is echogenic and its sonographic appearance is related to its dilution in the synovial fluid.

Figure 5A shows the needle within the MCP joint surrounded by highly echoic triamcinolone drops. Post-injection sonograms show complete filling of articular cavity by corticosteroid (fig 5B, C).

All the procedures required about $10 \mathrm{~min}-$ utes.

\section{Conclusion}

High frequency US allows a quick and detailed assessment of tendon and joint involvement in PA. In patients with clearly evident features of synovitis, infiltrative therapy under sonographic guidance is indicated. The usual technique of infiltrative therapy of finger joints uses
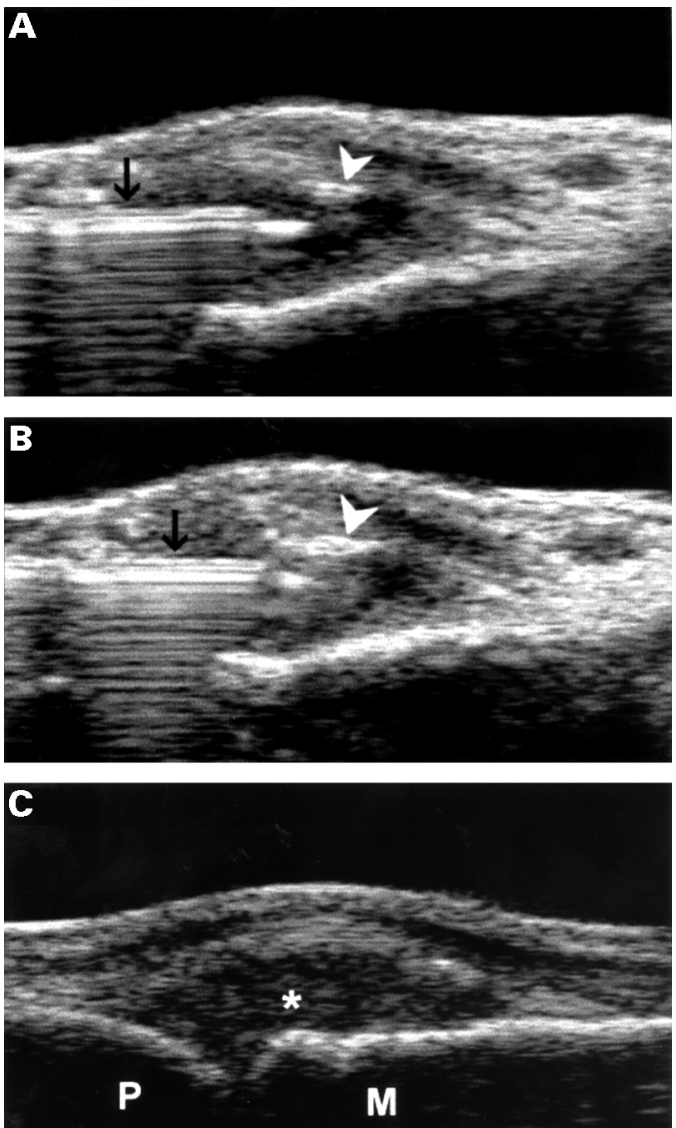

Figure 5 (A) Longitudinal sonogram of the MCP joint showing the placement of the tip of the needle within the joint cavity. The image has been obtained after the injection with a minimal amount of triamcinolone hexacetonide. Triamcinolone drops are highly echoic and can be easily visualised as white spots (arrowhead). Black arrow = needle. (B) Complete filling of the joint cavity by

corticosteroid. (C) Longitudinal sonogram of the MCP joint after the injection shows an echoic joint cavity ( $\left.{ }^{\star}\right) . M$ $=$ metacarpal bone; $P=$ proximal phalanx. 
a blind placement of the needle into the joint. This pictorial essay shows that sonographic guidance of needle position in MCP joint is a simple procedure, easy to perform, not time consuming and without damage to neighbouring structures.

Funding: this study was supported in part by Ministero della Università e della Ricerca Scientifica e Tecnologica (grant ref 30732 of 31.07.97), and by Consiglio Nazionale delle Ricerche (grant ref no 96.03037.CT04).

1 Grassi W, Tittarelli E, Pirani O, Avaltroni D, Cervini C Ultrasound examination of metacarpophalangeal joints in rheumatoid arthritis. Scand J Rheumatol 1993;22:243-7.

2 Grassi W, Tittarelli E, Blasetti P, Pirani O, Cervini C. Finger tendon involvement in rheumatoid arthritis. Evaluation with high-frequency sonography. Arthritis Rheum 1995; 38:786-94.

3 Grassi W, Cervini C. Ultrasonography in rheumatology: an evolving technique. Ann Rheum Dis 1998;57:268-71

4 Martinoli C, Derchi LE, Pastorino C, Bertolotto M, Silvestri E. Analysis of echotexture of tendons with US. Radiology 1993;186:839-43.

5 Manger B, Kalden JR. Joint and connective tissue ultrasonography. A rheumatological bedside procedure? A German experience. Arthritis Rheum 1995;38:736-42.

6 Lehtinen A, Taavitsainen M, Leirisalo-Repo M. Sonographic analysis of enthesopathy in the lower extremities of patients with spondylarthropathy. Clin Exp Rheumatol $1994 ; 12: 143-8$ 